\title{
Applications of Evolutionary Computation Techniques to Analog, Mixed-Signal and RF Circuit Design - An Overview
}

\author{
E. Roca ${ }^{1}$, M. Fakhfakh², R. Castro-López ${ }^{1}$, and F. V. Fernández ${ }^{1}$ \\ ${ }^{1}$ IMSE, CSIC and University of Sevilla \\ Sevilla, Spain \\ Email: Francisco.Fernandez@imse.cnm.es \\ ${ }^{2}$ University of Sfax \\ Sfax, Tunisia \\ Email: mourad.fakhfakh@ieee.org
}

\begin{abstract}
This paper reviews the application of evolutionary computation techniques to analog, mixed-signal and radiofrequency design problems. Design needs, limitations of existing approaches and open challenges are pointed out.
\end{abstract}

\section{INTRODUCTION}

Strategies to automate sizing of analog, mixed-signal and radio-frequency (AMS/RF) circuits have usually followed either a knowledge-based approach or an optimization-based approach. These strategies aim at finding the optimal sizing of the problem's variables that satisfies imposed constraints and maximize/minimize some objective functions, if any.

Knowledge-based approaches suffer from well-known limitations. Therefore, optimization-based approaches have become dominant for the past 15 years [1]. It is, however, important to note that the addition of AMS/RF design knowledge significantly enhances the optimization results by improving the design space exploration.

Optimization-based approaches formulate the design problem as a constrained optimization problem: minimize/maximize some objective functions (e.g., minimize power consumption, minimize area), subject to some constraints (e.g., slew rate larger than a certain value). This can be formulated as follows:

$$
\begin{aligned}
& \text { Minimize } \vec{f}(\vec{x}) ; \quad \vec{f}(\vec{x}) \in R^{k} \\
& \text { such that: } \\
& \vec{g}(\vec{x}) \leq 0 ; \\
& \vec{g}(\vec{x}) \in R^{m} \\
& \text { and } \vec{h}(\vec{x})=0 \text {; } \\
& \vec{h}(\vec{x}) \in R^{n} \\
& \text { where } x_{L i} \leq x_{i} \leq x_{U i}, \quad i \in[1, p]
\end{aligned}
$$

where $\vec{f}(\vec{x})=\left\{f_{1}(\vec{x}), f_{2}(\vec{x}), \ldots, f_{k}(\vec{x})\right\} \quad$ represents the $k$ objective functions (i.e., performance characteristics) to be

This work has been supported in part by the TIC-2532 Project, funded by Consejería de Innovación, Ciencia y Empresa, Junta de Andalucía, and in part by the Project TEC2007-67247/MIC funded by the Spanish Ministry of Science and Innovation with support from ERDF. minimized/maximized, and $\vec{g}(\vec{x})$ and $\vec{h}(\vec{x})$ are the inequality and equality constraints, respectively, that delimit the feasible region. Vector $\vec{x}$ corresponds to the $p$ design variables, and $\vec{x}_{L}$ and $\vec{x}_{U}$ contain their lower and upper bounds, respectively. If $k=1$, then the problem is a single-objective optimization problem; otherwise it is a multi-objective optimization problem.

Optimization algorithms can be classified into two major classes: exploration (global) and exploitation (local) algorithms. Exploitation approaches, which can be deterministic or not, are appropriate for fine-tuning of designs, but not for sizing starting from scratch. On the other hand, exploration algorithms are used in global sizing. Most famous ones are heuristics, and, probably, the most widely used heuristics in AMS/RF design have been genetic algorithms, simulated annealing and modified versions of them (e.g. [2],[3]).

In this paper, we will review some evolutionary computation (EC) techniques and their application to AMS/RF circuit design problems. Basic techniques are referenced in Section II and special attention is given to multi-objective problems in Section III. AMS/RF design problems are usually heavily constrained problems; hence, constraint handling mechanisms are addressed in Section IV and some current challenges are discussed in Section V.

\section{BASIC EVOLUTIONARY COMPUTATION TECHNIQUES}

Evolutionary Computation (EC) is a very active research area of computer science [4]. An important class of EC algorithms is Evolutionary Algorithms (EA), which are inspired from metaphors of biological processes. EAs encompass numerous approaches currently used in optimization, such as genetic algorithms, evolution strategies, etc. In short, an EA is a stochastic iterative procedure for generating tentative solutions for an optimization problem. The algorithm handles a set of individuals (candidate solutions), whose fitness (quality) determines the probability that they will be kept to be used as seeds for generating other individuals [4]. 
EAs have been successfully applied to solve circuit design problems [2], [5]-[9]. Despite this fact, the continuous evolution of technology imposes hard constraints on such algorithms which lead to many real difficulties, such as prohibitive evaluation cost (related with the size, complexity and fidelity of the circuit model), a large number of function evaluations, a large number of parameters to handle, etc.

Another class of nature-inspired EC algorithms, recently proposed, is swarm intelligence algorithms. They focus on animal behavior and insect conduct in order to develop some metaheuristics which can mimic their problem-solution abilities, namely Ant Colony (AC) [10], Artificial Bee Colony (ABC) [11], Wasp Nets [11] and Particle Swarm Optimization (PSO) [12]. The ability of these algorithms to solve AMS/RF design problems is also being investigated.

It is to be mentioned that some heuristic-based mathematical approaches have also been used to deal with AMS/RF optimization problems, such as local search [13], simulated annealing [14], and scatter search [15].

Traditionally, global optimization algorithms have been single-objective, i.e., they try to minimize/maximize a single objective function. More recently, algorithms specifically devoted to solve multi-objective optimization problems have become object of intensive research. Actually, solving real optimization problems, i.e., obtaining 'good' solutions in reasonable computation time while efficiently allocating computing resources, still presents numerous open challenges.

\section{MULTI-OBJECTIVE OPTIMIZATION}

Traditionally, multi-objective design problems have been addressed by applying a weighted sum of the objective functions, hence, transforming the multi-objective problem into a single-objective one. Then, any single-objective optimization technique can be applied. The weights of the different objectives intend to reflect the priority that the designer gives to some objectives over others. A first problem is that the weights assigned to the objectives do not necessarily map to the results obtained for each of them. But the major drawback is that it goes against the fundamental nature of multi-objective optimization. The intrinsic goal of multi-objective optimization is to provide the best trade-offs among the defined objectives, in the form of the so-called Pareto-optimal fronts (POF) [16]. To understand this concept, a few definitions are required.

A design point, $\vec{X}_{A}$, is said to dominate another design point, $\vec{x}_{B}$, (noted as $\vec{x}_{A} \prec \vec{x}_{B}$ ) if $\vec{f}\left(\vec{x}_{A}\right) \leq \vec{f}\left(\vec{x}_{B}\right)$ and $f_{i}\left(\vec{x}_{A}\right)<f_{i}\left(\vec{x}_{B}\right)$ for at least one function $i^{1}$. The design point $\vec{X}_{A}$ is said to be non-dominated if there is no other design that dominates it. The non-dominated set of the entire feasible performance space is known as the Pareto-optimal front. The concepts of dominance and Pareto-optimal front

\footnotetext{
${ }^{1}$ This formulation is valid for minimization problems. A simple change of sign applies for maximization.
}

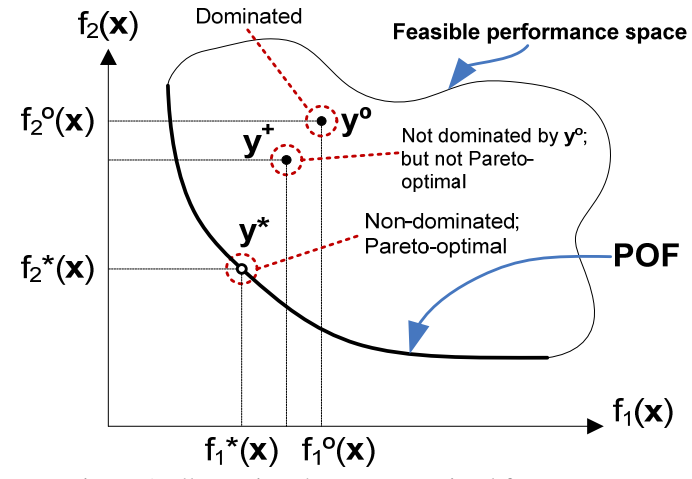

Figure 1. Illustrating the Pareto-optimal front concept.

are illustrated in Figure 1 for a two-dimensional performance space.

In fact, the weighted sum approach above corresponds to obtaining a single point of the POF in Figure 1. In principle, it is possible to generate the POF by repetitively applying the same approach with different values of the weights. One problem with this method is that it cannot provide solutions in possible non-convex parts of the Pareto front because a convex weighted sum of objectives converges only to the convex parts of the Front. For instance, for $k=2$, the optimization problem in (1) is transformed as follows:

$$
f_{e q}(\vec{x})=\omega_{1} f_{1}(\vec{x})+\omega_{2} f_{2}(\vec{x})
$$

As illustrated in Figure 2a, Equation (2) represents a straight line in the plane $\left(f_{1}, f_{2}\right)$. Thus minimizing $f_{e q}$ is nothing but minimizing the constant $c$ of equation (3):

$$
f_{2}(\vec{x})=-\frac{\omega_{1}}{\omega_{2}} f_{1}(\vec{x})+c
$$

It is clear, as it is shown in Figure 2b, that varying the weighting coefficients $\left(\omega_{1}, \omega_{2}\right)$ cannot allow sweeping the entire Pareto front. Besides, Figure 2c shows how much, in some cases, obtained solutions are sensitive to a small variation of these weightings [5]. Moreover, the weighting approach does not ensure, a priori, a homogeneous distribution of designs over the Pareto front. That is, a uniform assignment of weights does not mean that the Pareto front will be uniformly sampled. More importantly, as the number of samples increases, the approach becomes less efficient than the application of a truly multi-objective optimization algorithm.

In most cases, the POF generation problem in AMS/RF circuits has been addressed by means of evolutionary computation algorithms [2],[5]-[7]. An exception is the approach reported in [17], that makes use of the normal boundary intersection method. The biggest drawback of this method is that it might get stuck at local minima due to the optimization algorithms applied. An improvement, reported in [18], is to try to escape from local minima thanks to the exchange of solutions between the optimization algorithms that are executed in parallel. 


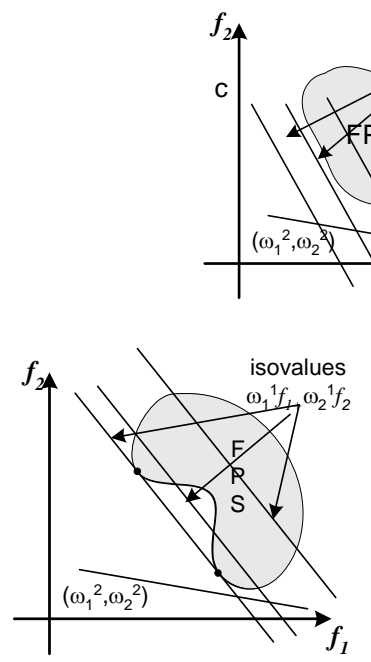

(b)

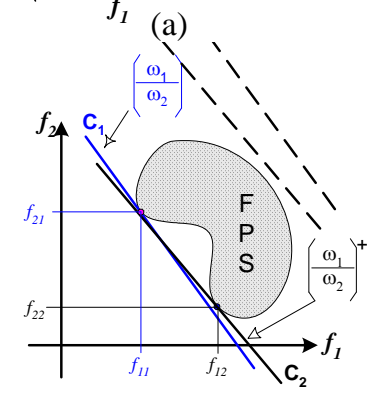

(c)
Figure 2. The weighting approach: principle and drawbacks.

\section{CONSTRAINT HANDLING}

AMS/RF design problems are usually heavily constrained optimization problems. The quality of the final results strongly depends on the mechanism used to handle constraints. Several approaches have been reported in the literature.

A first approach is to simply reject candidate individuals that do not meet the constraints. Though its implementation is very simple, it, however, exhibits an important drawback. On one hand, increasingly demanding performance specifications impose severe constraints to circuit sizing methods. On the other hand, the design variable space has usually a large number of dimensions. It infers from these two trends that the feasible design space can represent only a small region of the complete design space. It may therefore happen that when the initial population is sampled in the design space, no individual is feasible and, hence, the algorithm gets stuck.

Another widely used alternative is the static penalty function (PF) approach. In this method, the constrained optimization problem is transformed into an unconstrained one by minimizing the following function:

$$
f^{\prime}(x)=f(x)+W \cdot d(x, F)
$$

where $d(x, F)$ is a distance metric of the infeasible point to the feasible region $F$. It may simply be zero if no constraint violation occurs and is a positive number, otherwise. The definition of this distance metric includes the penalty coefficients that are used to stress the importance of a particular constraint violation over others. $W$ is a weight coefficient to set a global importance of the constraints with respect to the objective functions.

A major problem is that the results are sensitive to the values of the penalty coefficients and there is no general rule for setting them. A possible solution to palliate this problem has been published in [8], where the optimization problem is transformed into the minimization of an augmented
Lagrangian and a competitive co-evolution process automatically adapts the Lagrange multipliers.

One of the most effective techniques reported so far is the selection-based approach [19]. In this approach, the selection rules for a couple of candidate solutions in the population are:

1. Given two infeasible solutions, select the solution with smallest constraint violation,

2. If one solution is feasible and the other is not, select the feasible solution,

3. Given two feasible solutions, select that with best objective function value.

Precursor of this technique was the constraint handling mechanism in [3], essentially based on the same principle, although intended to compare a parent and a child solution, as the optimization algorithm was derived from simulated annealing. The selection mechanism is based on using a different cost function sign at the feasible and infeasible region.

The static penalty function approach can be easily extended to the multi-objective case by just adding a weighted sum of the objective function values to the fitness function. However, this approach suffers from the same drawbacks already discussed for the single-objective case.

The selection-based approach can be easily extended to the multi-objective case by just using a dominance comparison criterion instead of the objective function comparison. Candidate solutions obey the same selection aforementioned rules (the case of a single-objective problem), but when two solutions are feasible, respective objectives have to be compared:

1. if one of them dominates the other, choose the nondominated one;

2. if no solution dominates each other, choose one of them randomly.

\section{CHALLENGES}

Unlike the simple mathematical functions that are usually posed as benchmarks in the EC field, the fitness function evaluation in AMS/RF design problems is comparatively much more expensive, especially if a high level of accuracy is desired. Moreover, many design automation approaches are dealing with simulation-based optimization. Therefore, the investigation of more efficient algorithms that can provide a better trade-off of exploration (global search) and exploitation (local search) with fewer evaluations of the fitness function remains an important challenge.

Besides the constraint handling challenge already discussed, the efficient and accurate computation of the Pareto front constitutes a major challenge. Regarding constraints, and although problems of penalty function approaches are avoided, the calculation of the smallest constraint violation when both solutions are infeasible is still built as a weighted sum of the violation of each constraint. Experiments show that the convergence and extension of the POF may be heavily affected by the weights assigned to the constraint violations. 
An additional problem appears, especially in modern deep submicron technologies, due to the increasing random and systematic process variations. Process parameter variations map into variations of constraints and objectives, affecting the yield of manufactured circuits [1]. This problem may become especially dangerous in multi-objective optimization problems, because the effort of optimization algorithms to improve each objective as much as possible may severely increase the impact that process parameter variances pose on the objectives. That is, the obtained POF may be very good at the nominal design point but such point may be at the tail of the corresponding probability density function when parametric variations are considered. Therefore, incorporation of variability information in the optimization process becomes increasingly important [20]-[22]. Again, variability and yield assessment is a costly process and their incorporation must consider appropriate trade-offs of accuracy/efficiency on the fitness evaluation side (e.g., from the high-accuracy, low-efficiency of Montecarlo-based methods to the low-accuracy, high-efficiency of regressionbased methods) and more efficient search procedures on the optimization engine side.

In order to compare performances of different multiobjective optimization algorithms or even compare the sets of Pareto points from two independent runs of the same algorithm (e.g., with different algorithm parameter settings), appropriate POF quality evaluation metrics are needed [16]. POF evaluation and comparison criteria, which include different features of the POF, have been investigated extensively in the evolutionary computation (EC) field [16]. However, not all POF quality metrics seem to be suitable to analog design problems, and certainly not all needs of multiobjective analog synthesis are appropriately represented by the available metrics in the EC literature. Evaluation metrics basically address convergence and diversity. Most convergence metrics use the so-called true POF. Unlike mathematical benchmarks commonly used, the true POF is unknown in AMS/RF design problems. Moreover, the extent or a controlled non-uniformity of the POF may be important, aspects that have received little attention in the EC literature [23].

Most practical circuits are too complex to consider their design following a flat approach. Therefore, they require the application of hierarchical decomposition and design techniques. An important research area is the model of application of multi-objective optimization approaches in hierarchical problems. A model of application is the so-called multi-objective bottom-up optimization approach [9], in which the POF of the different blocks are composed bottomup across the different hierarchical levels. Some conditions must be met for such composition and a number of interconnection constraints arise. However, the POFs can also be used as feasibility hyper-surfaces for an optimal topdown hierarchical design approach.

\section{REFERENCES}

[1] G. Gielen, "CAD tools for embedded analogue circuits in mixed-signal integrated systems on chip,” IEE Proc. Comp. Digit. Tech., vol. 152, no. 3, pp. 317-332, 2005.
[2] M. Chu and D.J. Allstot, "Elitist nondominated sorting genetic algorithm based RF IC optimizer,” IEEE Trans. On Circuits and Systems - I, vol. 52. pp. 535-545, 2005.

[3] F. Medeiro et al., "Global design of analog cells using statistical optimization techniques,” Analog Integrated Circuits and Signal Processing, vol.6, pp.179-195, Nov. 1994.

[4] T. Weise, Global Optimization Algorithms - Theory and Applications. 2009, http://www.it-weise.de.

[5] M. Fakhfakh et al., "A novel heuristic for multi-objective optimization of analog circuit performances,” Analog Integrated Circuits and Signal Processing, vol. 61, no. 1, pp. 47-64, 2009.

[6] T. Eeckelaert, T. McConaghy and G. Gielen, "Efficient multiobjective synthesis of analog circuits using hierarchical Pareto-optimal performance hypersurfaces,” Proc. of the Design Automation and Test in Europe Conference, Mar. 2005.

[7] R. Castro-López, E. Roca, and F.V. Fernández, "Multimode Pareto fronts for design of reconfigurable analogue circuits," Electronics Letters, vol. 45, no. 2, pp. 95-97, Jan. 2009.

[8] B. Liu, Y. Wang, Z. Yu, L. Liu, M. Li, Z. Wang, J. Lu and F. V. Fernández, "Analog circuit optimization system based on hybrid evolutionary algorithms,”, Integration-the VLSI Journal, vol. 42, no. 2, pp. 137-148, Feb. 2009.

[9] T. Eeckelaert, R. Schoofs, M. Steyaert, G. Gielen and W. Sansen, “A continuous-time delta-sigma modulator for $802.11 \mathrm{a} / \mathrm{b} / \mathrm{g}$ WLAN implemented with a hierarchical bottom-up optimization methodology," Analog Integrated Circuits and Signal Processing, vol. 55, pp. 37-45, Jan. 2008.

[10] M. Dorigo, G. DiCaro and L. M. Gambardella, “Ant algorithms for discrete optimization,” Artificial Life Journal, vol.5, pp. 137-172, 1999.

[11] F. T. S. Chan and M. K. Tiwari, Swarm Intelligence: Focus on Ant and Particle Swarm Optimization, I-Tech Publishing. December 2007.

[12] J. Kennedy, R.C. Eberhart, "Particle swarm optimization,” Proc. IEEE Int. Conf. on Neural Networks. WA, Australia, pp 1942-1948, 1995.

[13] E. Aarts and K. Lenstra, Local Search in Combinatorial Optimization. Princeton University Press, 2003.

[14] R. Rutenbar, "Simulated annealing algorithms: an overview," IEEE Circuits and Devices Mag., pp. 19-26, 1989.

[15] M. Laguna and R. Martí, Scatter Search: Methodology and Implementation in C. Series Operations Research/Computer Science Interfaces Series, vol. 24, Springer, 2003.

[16] K. Deb, Multi-objective Optimization Using Evolutionary Algorithms. John Wiley \& Sons, 2001.

[17] G. Stehr, H. Graeb and K. Antreich, “Analog performance space exploration by normal-boundary intersection and by Fourier-Motzkin elimination,” IEEE Trans. on Computer-aided Design, vol. 26, no. 10, pp. 1733-1748, Oct. 2007.

[18] D. Mueller, H. Graeb and U. Schlichtmann, “Trade-off design of analog circuits using goal attainment and wave front sequential quadratic programming," Proc. Design, Automation and Test in Europe Conference, pp.75-80, Apr. 2007.

[19] K. Deb, "An efficient constraint handling method for genetic algorithm,” Computer Methods in Applied Mechanics and Engineering, vol. 186, pp. 311-338, 2000.

[20] H. Graeb, D. Mueller and U. Schlichtmann, "Pareto optimization of analog circuits considering variability,” Proc. European Conf. Circuit Theory and Design, pp. 28-31, 2007.

[21] T. McConaghy et al., "Variation-aware structural synthesis of analog circuits via hierarchical building blocks and structural homotopy,” IEEE Trans. Computer-Aided Design, vol. 28, pp. 1281-1294, Sep. 2009.

[22] S. Tiwary, P. Tiwary and R. Rutenbar, "Generation of yield-aware Pareto surfaces for hierarchical circuit design space exploration,” Proc. ACM/IEEE Design Automation Conf., pp. 24-28, 2006.

[23] F.V. Fernández, B. Liu, R. Castro-López and E. Roca, "Quality metrics of Pareto-optimal fronts for multi-objective synthesis of analog ICs," Proc. Int. Workshop on Symbolic and Numerical Methods, Modeling and Applications to Circuit Design”, pp. 56-60, 2008. 